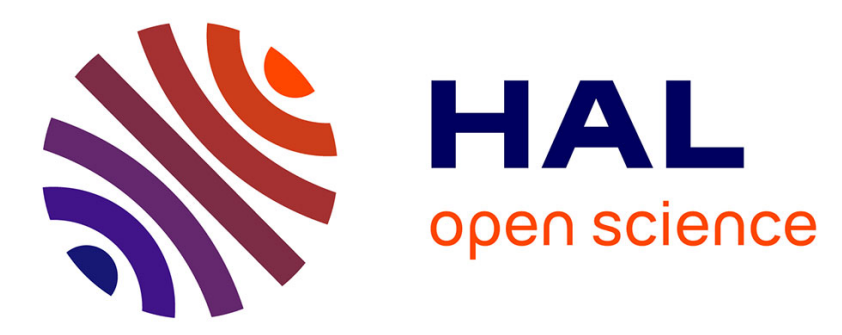

\title{
Simulation of grain evolution in solidification of silicon on meso-scopic scale
}

Xiaofang Qi, Lijun Liu, Thècle Riberi-Béridot, Nathalie Mangelinck-Noel, Wolfram Miller

\section{- To cite this version:}

Xiaofang Qi, Lijun Liu, Thècle Riberi-Béridot, Nathalie Mangelinck-Noel, Wolfram Miller. Simulation of grain evolution in solidification of silicon on meso-scopic scale. Computational Materials Science, 2019, 159, pp.432-439. 10.1016/j.commatsci.2018.12.015 . hal-02008484

\section{HAL Id: hal-02008484 \\ https://hal.science/hal-02008484}

Submitted on 27 Feb 2019

HAL is a multi-disciplinary open access archive for the deposit and dissemination of scientific research documents, whether they are published or not. The documents may come from teaching and research institutions in France or abroad, or from public or private research centers.
L'archive ouverte pluridisciplinaire HAL, est destinée au dépôt et à la diffusion de documents scientifiques de niveau recherche, publiés ou non, émanant des établissements d'enseignement et de recherche français ou étrangers, des laboratoires publics ou privés. 


\title{
Simulation of grain evolution in solidification of silicon on meso-scopic scale
}

\author{
Xiaofang $\mathrm{Qi}^{1,2}$, Lijun $\mathrm{Liu}^{2}$, Thècle Riberi-Béridot ${ }^{3}$, Nathalie \\ Mangelinck-Nö̈ ${ }^{3}$, and Wolfram Miller ${ }^{1, *}$ \\ Leibniz Institute for Crystal Growth (IKZ), Max-Born-Str. 2, 12489 Berlin, Germany \\ School of Energy and Power Engineering, Xi'an Jiaotong University, 710049 Xi'an, \\ China \\ 3 Aix Marseille Univ, Université de Toulon, CNRS, IM2NP, 13397 Marseille, France
}

\begin{abstract}
We present a cellular automata model for computing the grain evolution during directional solidification of silicon on a meso-scopic scale. Firstly, the method is applied to test cases with different shapes of the melt/crystal interface. In a second step we compute the case of an experiment with in-situ observation of the interface shape evolution [1]. Here we also include the effect of twinning. The interchanging appearance of two twins could be revisited by our calculations. The probabilities used correspond to those which were analytically derived for an undercooling of $\Delta T=0.6 K[29]$. This undercooling is a typical value for a groove with $\langle 111\rangle$ facets [34].

Keywords: Directional Solidification; Multicrystalline Silicon; Cellular Automata; Lattice Boltzmann Methods;
\end{abstract}

\section{Introduction}

Grain evolution is one of the key issues during the directional solidification of multi-crystalline silicon (mc-Si) for photovoltaic applications, because the final grain structure and related defects of silicon ingots strongly influence the conversion efficiency of solar cells [2]. Generally, defects such as grain boundaries $(\mathrm{GBs})$, impurity segregation and dislocations prevent the formation of homogeneous grain structure of mc-Si ingots. Once a mc-Si ingot is obtained by casting, it is hard to drastically change its grain structure in the solid state. Therefore, it is necessary to gain a better understanding of the grain evolution during the solidification process and thus control the final grain arrangement. For this reason, fundamental studies for the grain growth have come into focus recently.

Generally, grain competition is one of the key mechanisms for the evolution of the grain structure. Influence of growth conditions on the grain competition in mc-Si has been investigated from both experimental and theoretical viewpoints 
$[3,4,5]$. More specifically, the shape of GB groove at the triple junction of grain-grain-melt is the determining factor for which grain will grow faster than the others in the solidification growth, as explained by theoretical studies [6] and simulation results [7]. In the latter numerical studies it was also shown that the temperature field around a groove adapt to the situation of strongly curved melt/solid interfaces and growth kinetics. The temperature gradient in the groove and its vicintiy is different from the avarage one at the interface.

On the other hand, changes of grain structure will also occur due to the GBs interaction in the growth process. GB interactions during the growth process include GB generation and annihilation, which will lead to variation of the total number of GBs, grain sizes and GB types. Experimental research revealed that the majority of GBs in pure samples originated from $\Sigma 3$ twins ( $\Sigma$ boundaries are boundaries where atoms of the two grain coincides after a certain period), and that the interaction between $\Sigma$ twinned grains is dominant during the grain evolution process $[8,9,10]$. On the microscopic scale, phase field methods have been applied to compute grain growth [11]. Phase field methods are an important technique for investigating the details of grain evolution in direct solidification of Si including the effects of interface curvature but they are quite time consuming and thus unable to calculate the soldification on the typical time scale for silicon (hours).

Despite of the ongoing investigations of the grain growth during the solidification of mc-Si, the work on the modeling and simulation of the grain evolution on the macroscopic scale is still a challenge due to the complexity of multi-scale modeling. Some attempts have been made to study the grain structure on the macroscopic scale $[12,13]$, in which only simplified geometrical rules for the evolution of GBs were applied. Cellular automata (CA) methods are widely used for computation of grain evolution $[14,15]$. Rappaz et al. were the first using this kind of methods to predict the solidification of grain structures [16]. Recently, several researchers have been simulated the nucleation and crystalline structure formation for mc-Si by employing $\mathrm{CA}$ and finite element models in the commercial software ProCAST $[17,18,19]$. CA are mesoscale methods, which can efficiently bridge the gap between micro- and macroscopic scale calculations, allowing the simulation of a large-scale crystal growth. However, the CA method usually ignores some details in the microscopic simulation method, such as anisotropy and faceting.

We have developed a two dimensional (2D) model, which uses input data based on results of calculation on the microscopic scale, taking into account the details of growth kinetics and interfacial energy effects $[3,7]$. The phase transition is computed by using a sharp interface model and a CA method is employed to calculate the grain orientation. We are going beyond the typical approaches of CA methods and take into account results of microscopic calculations and of dedicated experiments to set the rules of grain growth competition. Microscopic calculations in this contexts mean computations covering the details in the groove and at the multiple phase junctions between melt and grains including the curvature effects on undercooling. We perform such computations by employing the phase field method introduced by Kobayashi et al. [20] (see 


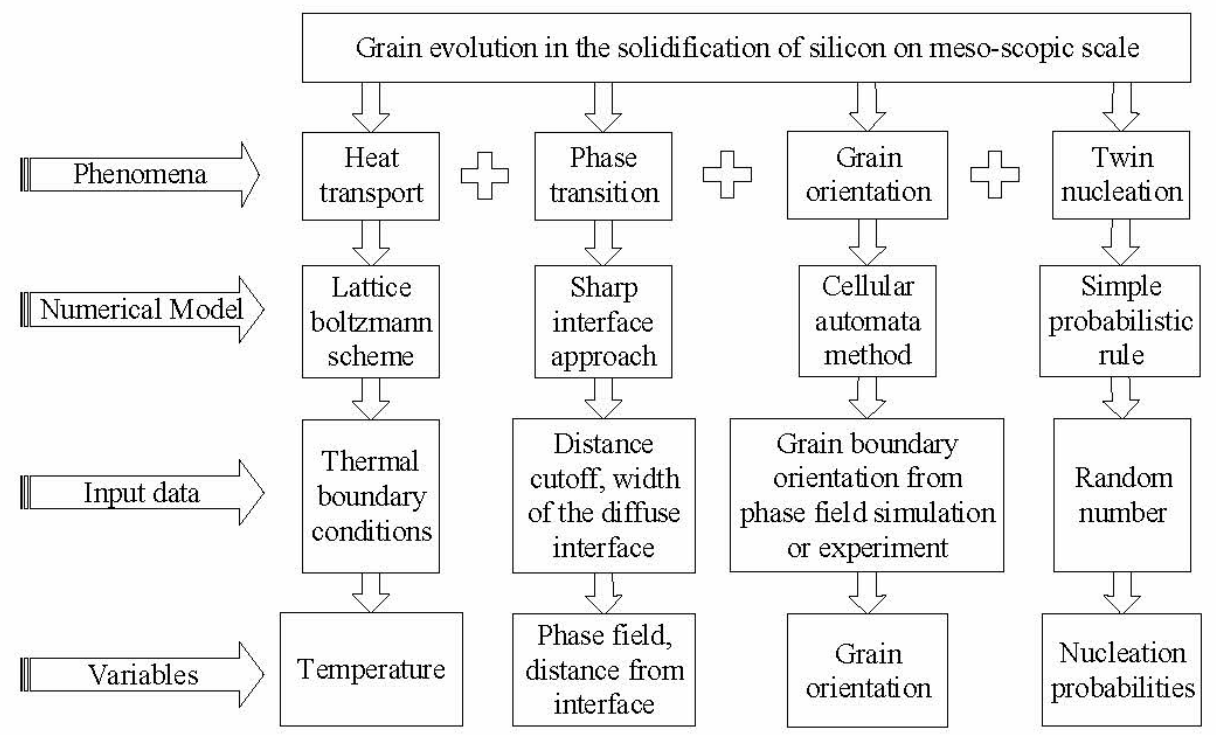

Figure 1: Schematic of different phenomena, numerical model, input data and variables for grain evolution.

e.g. [7]). In particular, the input data consists of the GB orientations with respect to the interface normal for every combination of GBs, are taken from microscopic calculations or dedicated experimental results. By using this approach combining $\mathrm{CA}$, a sharp interface model, and input data, we can consider effects interfacial energies, growth kinetics and their anisotropies in large scale simulations. As a preliminary example, we restrict our computations to 2D cases and investigate the influence of the crystal/melt interface shape on the grain evolution. Furthermore, the numerical model is extended to calculate the nucleation of twin seeds at the crystal/melt interface. This model enables us to compute the grain evolution as observed in experiments with in-situ X-ray imaging observation [9].

\section{Numerical Method}

The keypoint of the algorithm is the computing of the phase transition by a sharp interface approach on a fixed grid, i.e. the interface is located between grid points, and a cellular automata (CA) for updating the grain orientation. The rules for the CA are kept simple but complex enough to cover the relevant physics at three phase junctions of melt and two grains. The input parameters for the rules are taken from the results of microscopic calculations if possible or they are determined from experimental results. A simple probabilistic rule for twin nucleation is also introduced. Fig. 1 shows schematic of different phenomena, numerical model, input data and variables for grain evolution. 


\subsection{Phase transition and heat transport}

We use a hybrid algorithm for computing the phase transition. A sharp interface is used on off-grid points and the movement of the interface is computed by a kinetic operator (see e.g.[21]) to treat the growth kinetics at $\langle 111\rangle$ facets. The computation of the interface movement is done node-wise and the calculation is local. In order to correlate neighbouring nodes we additionally use a phase field $\phi$. The gradient of the phase field $\nabla \phi$ is computed by applying a nine-point stencil finite difference method using all lateral and diagonal neighbours (see e.g. [22]). This ensures the coupling of nodes with respect to the the position of the interface. In particular, the computation of the interface follows these two steps:

Step 1 If the distance at node $\vec{x}(i, j, k)$ to interface is smaller than a cutoff $\left(10^{-5} \mathrm{~m}\right)$, the phase field is updated:

(a) Compute the growth direction by $\nabla \phi$.

(b) Compute the temperature at interface $T_{\text {int }}$ using distance $\tilde{x}(i, j, k)$ and $\vec{n}=\nabla \phi /|\nabla \phi|$.

(c) Compute the growth velocity $v_{n}$ using $T_{\text {int }}$ and a kinetic operator.

(d) Compute new distance from interface $\tilde{x}(t+\delta t)=\tilde{x}(t)+\left|v_{n}\right| \delta t$.

(e) Compute new $\phi: \phi(t+\delta t)=\frac{1}{2}\left\{1+\tanh \left(\frac{3 \tilde{x}(t+\delta t)}{\delta}\right)\right\}$, where $\delta$ is the width of the diffuse interface.

Please note that the diffuse interface is only used for computing the interface normal.

Step 2 Update $\phi$ in cells, where Step 1 was not done but in one of the neighbouring cells.

$T_{\text {int }}$ is computed at an off-lattice point. In the following the details of computing are given taking into account the different thermal conductivities in melt and solid. The geometrical situation is shown in Fig. 2 (a).

The point $\vec{x}_{\text {int }}$ on the interface of node $\vec{x}(i, j, k)$, i.e. $\vec{x}_{\text {int }}=\vec{x}(i, j, k)+$ $\tilde{x}(i, j, k) \vec{n}$, lies within a quadrat bounded by iwest, ieast, jnorth, and $j$ south. The interface line will cross two boundary lines (see Fig. 2 (a)). The temperature at the crossing points $x_{c 1}$ and $x_{c 2}$ will be computed according to

$$
T_{\mathrm{int}}\left(x_{c i}\right)=\frac{D_{T}^{\mathrm{s}} \Delta x_{s} T(\vec{x} @ \phi<0.5)+D_{T}^{\mathrm{m}} \Delta x_{m} T(\vec{x} @ \phi \geq 0.5)}{D_{T}^{\mathrm{s}} \Delta x_{s}+D_{T}^{\mathrm{m}} \Delta x_{m}},
$$

where $\vec{x} @ \phi<0.5$ and $\vec{x} @ \phi \geq 0.5$ are the nodes in the liquid and solid part, respectively. The temperature at the interface used for the computation of the interface velocity is given by:

$$
T_{\mathrm{int}}=\frac{T_{\mathrm{int}}\left(x_{c 1}\right)\left|\vec{x}_{c 2}-\vec{x}_{\mathrm{int}}\right|+T_{\mathrm{int}}\left(x_{c 2}\right)\left|\vec{x}_{c 1}-\vec{x}_{\mathrm{int}}\right|}{\left|\vec{x}_{c 2}-\vec{x}_{c 1}\right|} .
$$


(a)

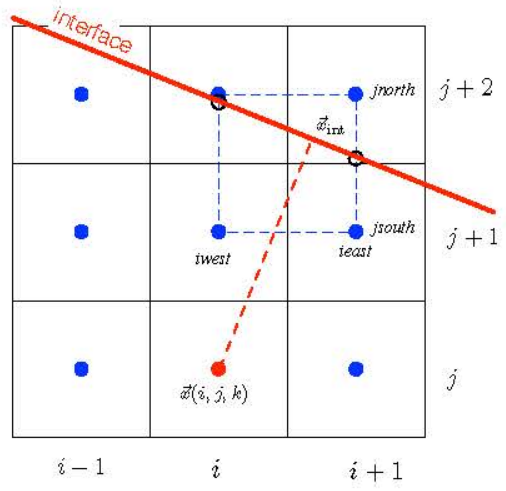

(b)

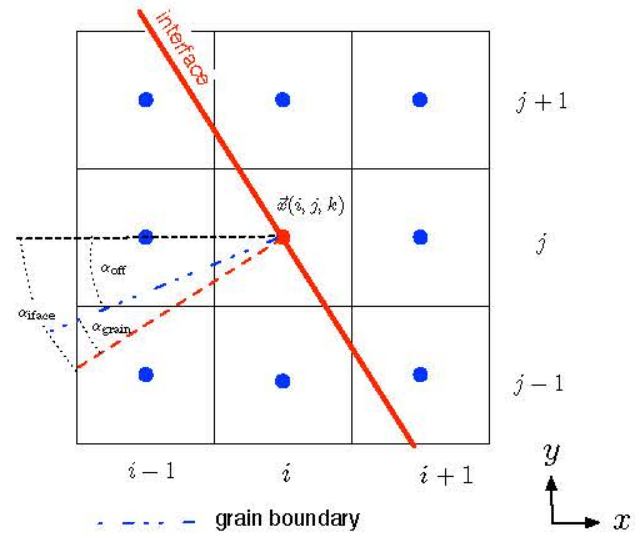

Figure 2: Left (a): Sketch for computing the temperature at the interface for using it to calculate the interface velocity. Right (b): Definition of angles for updating grain orientation.

The heat transport equation

$$
\frac{\partial T}{\partial t}=\nabla\left(D_{T} \nabla T\right)+S_{L}
$$

is solved by a Lattice Boltzmann scheme [23]. $S_{L}$ is the latent heat soruce, which is defined as follows:

$$
S_{L}=\frac{\partial \phi}{\partial t}=\frac{\Delta H_{V}}{\rho c_{p}}
$$

where $\Delta H_{V}, \rho, c_{p}$ are the latent heat per volume, the density, and the specific heat, respectively.

The thermal diffusivity at node $\vec{x}(i, j, k)$ is set by:

$$
D_{T}(i, j, k)=D_{T}^{\mathrm{s}} \phi(i, j, k)+D_{T}^{\mathrm{m}}(1-\phi(i, j, k)),
$$

where $D_{T}^{\mathrm{s}}$ and $D_{T}^{\mathrm{m}}$ are the thermal diffusivities in solid and melt, respectively.

\subsection{Updating grain orientation}

The computational domain in our calculation is a rectangle with a uniform grid. Every grain is given a number and this grain number is unique in a cell. In other words, there the grain orientation is the same in the entire cell and a grain boundary is located on the boundary of the cells and is not off-grid as the interface line. To begin with, we set an artificial initial crystal/melt interface shape and a configuration of grains. In the melt there is no grain orientation and the grain number is set to -1 .

We now consider a node at time $t$ with a phase field value $\phi(t)<0.45$. If $\phi(t) \geq 0.45$ in the next time step $(t+1)$ a grain number has to be attributed 


\begin{tabular}{lcccc}
\hline Material parameter & & Unit & Value & \\
\hline Thermal diffusivity in melt & $D_{T}^{m}$ & $\mathrm{~m}^{2} \cdot \mathrm{s}^{-1}$ & $2.64 \times 10^{-5}$ & {$[24]$} \\
Thermal diffusivity in solid & $D_{T}^{s}$ & $\mathrm{~m}^{2} \cdot \mathrm{s}^{-1}$ & $0.9 \times 10^{-5}$ & {$[25]$} \\
Latent heat & $\Delta H_{V}$ & $\mathrm{~J} \cdot \mathrm{m}^{-3}$ & $4.22 \times 10^{9}$ & {$[26]$} \\
Specific heat & $\rho c_{p}$ & $\mathrm{~J} \cdot \mathrm{K}^{-1} \cdot \mathrm{m}^{-3}$ & $2.2 \times 10^{6}$ & {$[27]$} \\
Melting-point temperature & $T$ & $\mathrm{~K}$ & 1683 & \\
\hline
\end{tabular}

Table 1: Thermophysical properties of Si used in simulations.

to this node. In order to do so we consider the grains at three adjacent nodes. The choice of the three nodes depends on the local curvature of the interface as depicted in Fig. 3. If $\alpha_{\text {iface }}$ (see Fig. 2 (b)) is between $-45^{\circ} / 2$ and $+45^{\circ} / 2$ we use the three nodes at $i-1$ (Fig. 3 (a)). If $45^{\circ} / 2<\alpha_{\text {iface }}<45^{\circ}+45^{\circ} / 2$ we use the nodes as indicated in Fig. 3 (b). If $45^{\circ}+45^{\circ} / 2<\alpha_{\text {iface }}<90^{\circ}$ we use the nodes as indicated in Fig. 3 (c). Currently, we do not allow a growing in negative $\mathrm{x}$-direction. For cases (b) and (c) in Fig. 3 there exist also the corresponding cases with growth direction in $+\mathrm{y}$ direction and nodes 1,2 or $0,1,2$ at $j-1$.

When a node $\vec{x}(i, j, k)$ changes from the melt region to solid region in the solidification process, we firstly choose the selection scheme according to the interface normal, as shown in Fig. 3. Then, if all the three neighbour nodes have the same grain number, i.e. belong to the same grain, also the node $\vec{x}(i, j, k)$ belongs to this grain and thus, this grain number is set to the node. If the grains are different, the orientation of GB has to be computed. As the node $\vec{x}(i, j, k)$ has a unique value of grain number, we need a set of rules in the manner of a CA to compute the new value. For every combination of GBs in a run, $\alpha_{\text {grain }}$ is stored prior to computation. Please note that the orientation of the grains is measured against the interface normal (dotted red line in Fig. 2). This means that for the same combination of two grains in the meso-scopic calculation $\alpha_{\text {grain }}$ depends on $\alpha_{\text {iface }}$ and might be quite different in time. As $\alpha_{\text {iface }}$ was computed from the phase field and $\alpha_{\text {grain }}$ is picked up from the input table for the particular $\alpha_{\text {iface }}, \alpha_{\text {off }}$ can be computed via

$$
\alpha_{\text {off }}=\alpha_{\text {grain }}+\alpha_{\text {iface }}
$$

The values for $\alpha_{\text {grain }}$ can be determined from experimental results or calculated on microscopic scale as e.g. by phase field simulation [3, 7]. Once $\alpha_{\text {off }}$ has been determined, one can compute the probabilities that the node $\vec{x}(i, j, k)$ becomes one or the other grain. The choice is done via a random generator. By definition of the CA the grain boundary is a zig-zag line following the grid with an average inclination equivalent to $\alpha_{\text {off }}$. This has been tested for the evolution of a planar interface.

There might be also locally a back melting of the solid. When $\phi(t)$ at a node becomes smaller 0.1 the grain number at this node is set to unknown. Once this node becomes solid again, the grain orientation has to be recomputed. 


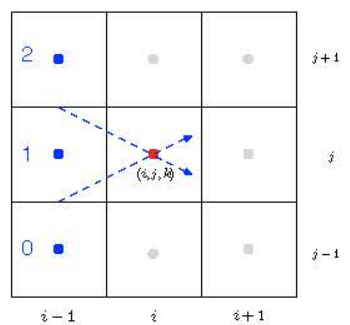

(a)

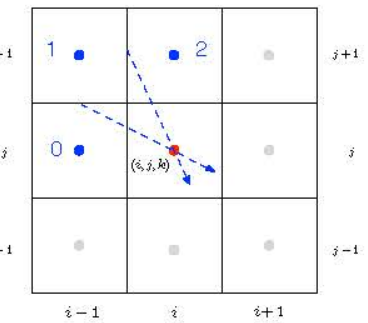

(b)

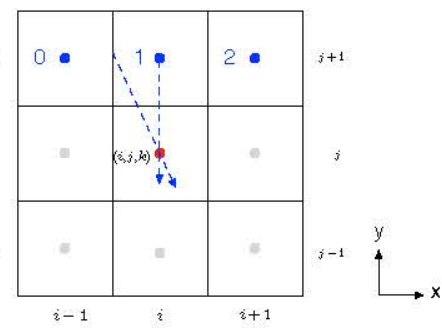

(c)

Figure 3: Grain selection schemes in the solidification process according to the curvature of the crystal/melt interface shape. We distinguish three cases depending on the interface shape. The blue dotted lines indicate the extreme values for the interface normals for the case. The blue nodes are the nodes used for computing the grain at the red point $(i, j, k)$. Case (a): $-\frac{45^{\circ}}{2} \geq \alpha_{\text {iface }} \geq \frac{45^{\circ}}{2}$. Case (b): $\frac{45^{\circ}}{2}<\alpha_{\text {iface }}<45^{\circ}+\frac{45^{\circ}}{2}$. Case (c): $45^{\circ}+\frac{45^{\circ}}{2}<\alpha_{\text {iface }}$. For cases (b) and (c) there exist the corresponding cases in -y direction.

\subsection{Nucleation of twin seeds}

Twin nucleation occurs frequently during the growth process of mc-Si. The mechanism of twin nucleation for mc-Si crystal growth was proposed by Duffar et al. [28] and modified recently by Lan et al. [29]. The nucleation occurs at grooves with at least one $\langle 111\rangle$ facet. Then a $\Sigma 3$ coherent twin boundary is formed. The probability of nucleation is given as a function of the local undercooling. On the meso-scopic scale we use such a probability for grain boundaries with facetted/facetted grooves. Experimetally, twin nucleation and subsequent creation of a $\Sigma 3$ coherent twin boundary was e.g. by Riberi et al. [9]. In this paper we restrict ourselves to describe the situation for the experiment described in [9].

When a cell becomes solid ( $\phi$ becomes larger than 0.45 ) we apply a random generator and check if the random number is smaller than the (fixed) probability for nucleation. For a facetted/facetted groove nucleation can occur on one of the facets. In the experiment of [9] the nucleation always occured on the same side of the groove and thus we also take this side in our numerical calculations. Because it is twinning the orientation of the new grain is given by the one of the grain with the facet at the groove. The probabilities for nucleation is deduced from the experimental results, directly from the frequence of the occurence of a new twin boundary in the experiment.

\subsection{High performance computation}

The algorithms have been implemented into the software environment WaLBErla [30, 31]. WalBErla is an object oriented toolbox, which ensures a high performance in massively parallel computing. The random generator of the standard C-Library was used. As the update of the grains is done via Monte Carlo procedure, we performed always several runs for a specific case choosing different initializations of the random generator. The resulting grain structures were comparable and we will show only the results of one run per case. In 

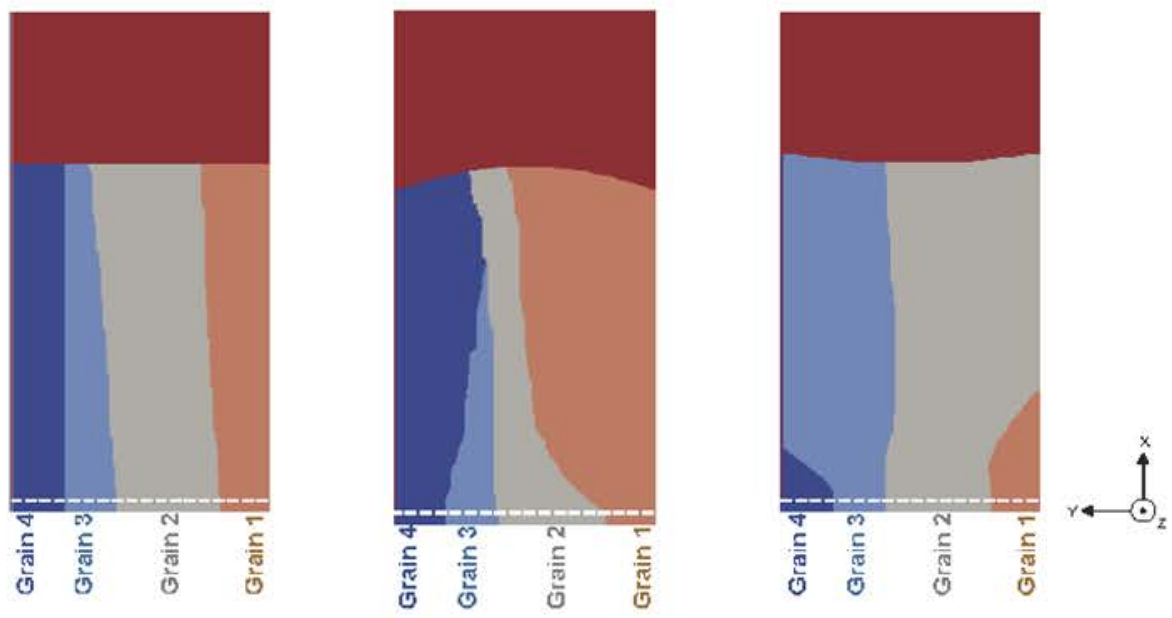

Figure 4: Evolution of grains for different crystal/melt interface shapes at $80 \%$ solidification. The angles between the main growth direction and the $\langle 100\rangle$ orientation are $0^{\circ},-10^{\circ}, 5^{\circ}$, and $-5^{\circ}$ for Grain $1,2,3$, and 4 , respectively. (a) Flat, (b) convex and (c) concave interface shape.

particular, for the cases discussed in this paper, the relative difference in area fraction of the various grains is less than $2 \%$.

\section{Calculations}

\subsection{Domain and thermal conditions}

In all calculations we use a rectangular domain containing the silicon. No other parts of the furnace are currently included in the computations and realistic thermal boundary conditions need to be set. We try to mimic the typical process in a solidification experiment. In the real experiment the temperature is continuously reduced according to a cooling rate $\mathcal{R}_{\text {cool }}$. This cooling rate is applied at the bottom boundary $(x=0)$. The temperature gradient in the melt $G_{T}^{m}$ is initially defined by setting the temperature at the top boundary via $T_{\mathrm{bc}}(t=0)=T_{m}+G_{T}^{m} x_{\text {int }}$, where $x_{\text {int }}$ is the position of the interface at $t=0$. The temperature at time $t$ is given by $T_{\mathrm{bc}}(t)=T_{\mathrm{bc}}(t=0)-\mathcal{R}_{\text {cool }} t$. The thermal boundary condition at bottom is set as follows: the incoming heat flux at $t=0$ is given by $\lambda^{m} G_{T}^{m}$ ( $\lambda^{m}$ is the heat conductivity in the melt). The outgoing heat flux corresponds to the incoming flux plus the one due to the latent heat production. The latter is given by $v_{n}^{\text {theor }} \Delta H_{V}$, where $v_{n}^{\text {theor }}$ is the theoretical growth velocity deduced from $v_{n}^{\text {theor }}=\mathcal{R}_{\mathrm{cool}} / G_{T}^{m}$. Thus, the temperature gradient in the solid $G_{T}^{s}$ is in a thermally steady-state situation:

$G_{T}^{s}=\frac{\lambda^{m}}{\lambda^{s}} G_{T}^{m}+\frac{\Delta H_{V}}{\lambda^{s}} \frac{\mathcal{R}_{\mathrm{cool}}}{G_{T}^{m}}=\frac{D_{T}^{m} \rho^{m} c_{p}^{m}}{D_{T}^{s} \rho^{s} c_{p}^{s}} G_{T}^{m}+\frac{\Delta H_{V}}{D_{T}^{s} \rho^{s} C_{p}^{s}} \frac{\mathcal{R}_{\mathrm{cool}}}{G_{T}^{m}}=\frac{D_{T}^{m}}{D_{T}^{s}} G_{T}^{m}+\frac{\Delta H_{V}}{D_{T}^{s} \rho c_{p}} \frac{\mathcal{R}_{\mathrm{cool}}}{G_{T}^{m}}$. 

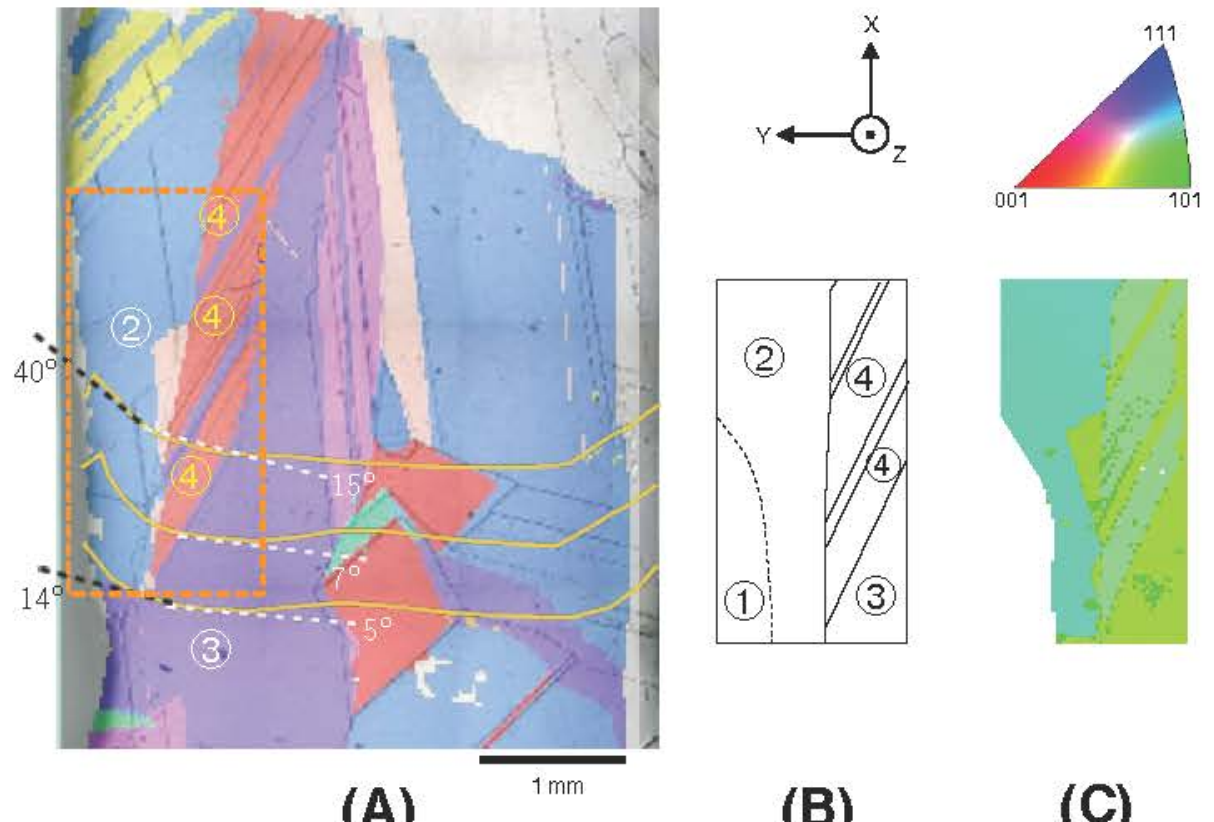

(B)

(C)

Figure 5: Experimental result of Riberi et al. [9]. (A) EBSD map in x-direction with interface lines (in yellow) from in-situ observation. (B) Part with simplification, which is revisited in numerical calculations. (C) EBSD map in z-direction, showing that all grains taken into account are almost oriented with the (101) plane in the cut. The labels (1) to (4) denote the different grains used in the numerical calculations.

Here, we assume $\rho^{m} c_{p}^{m} \equiv \rho^{s} c_{p}^{s}=\rho c_{p}$ for convenience ${ }^{1} . D_{T}, \rho, c_{p}$ are the thermal diffusivity, the density, and the heat capacity, respectively. $m$ and $s$ denote melt and solid, respectively. Thus, we set the heat flux at bottom according to Eq. 7:

$$
j=D_{T}^{m} \rho c_{p} G_{T}^{m}+\Delta H_{V} \frac{\mathcal{R}_{\mathrm{cool}}}{G_{T}^{m}} .
$$

\footnotetext{
${ }^{1}$ In the vicinity of the melting point $\rho^{5} c_{p}^{5}=2.45 \times 10^{6} \mathrm{~J} \mathrm{~m}^{-3} \mathrm{~K}^{-1}$ using data of [32]. For melt one has $\rho^{s} c_{p}^{s}=2.65 \times 10^{6} \mathrm{~J} \mathrm{~m}^{-3} \mathrm{~K}^{-1}$ using data of [24] and [33] or $\rho^{s} c_{p}^{s}=2.2 \times$ $10^{6} \mathrm{~J} \mathrm{~m}^{-3} \mathrm{~K}^{-1}$ when using the more recent data of [27]. Thus, the experimental uncertainties are of the same level as the difference in $\rho^{m} c_{p}^{m}$ and $\rho^{s} c_{p}^{s}=\rho c_{p}$.
} 


\subsection{Effects of the interface shape}

Firstly, we studied the effect of the crystal/melt interface shape on the grain evolution in the solidification process with the given cooling rate and temperature gradient in the melt. As shown in Fig. 4, the computational domain is $25 \times 10 \mathrm{~mm}^{2}$ descretized with a uniform grid with a spacing of $\Delta x=5 \times 10^{-5} \mathrm{~m}$. The time step is set to $\Delta t=1.0 \times 10^{-3} \mathrm{~s}$. The initial crystal $/$ melt interface (in white dotted lines) is located at $x=5 \times 10^{-4} \mathrm{~m}$. Four grains are set at the bottom and grains grow towards upward ( $+\mathrm{x}$-direction). The orientation variable is assigned to each grain, and the initial angles between the $\langle 100\rangle$ orientation and the main growth direction are $0^{\circ},-10^{\circ}, 5^{\circ}$, and $-5^{\circ}$ for grains 1 , 2, 3, and 4, respectively. Fig. 4 shows the evolution of the grain structure under different crystal/melt interface shapes when the solidification fraction is $80 \%$. An additional heat flux is set to the side boundaries of the domain to control the interface shape. The simulated results reveal that the crystal $/$ melt interface shape significantly influences the grain evolution and grain distribution during the solidification process. For example, the crystal/melt interface shape is totally flat in Fig. 4(a) when heat fluxes at both side boundaries are zero. The GBs are almost straight lines for the flat interface shape in Fig. 4(a). While, for the convex and concave interface shapes, the GBs gradually change direction according to the orientation of the crystal/melt interface. As shown in Fig. 4(b), grains 2 and 4 gradually extend laterally, and finally stop the progress

of grain 3, resulting in the formation of a new grain boundary. In the case of concave interface grains 2 and 3 prevail whereas grains 1 and 4 dissapear after a short time.

Please note that here we use some artifical input data for the grain boundary orientation as a function of the growth direction. An input based on phase field simulations will be presented in the second example. It is evident that both interfacial energies at the three phase junction and local growth kinetics determine the grain competition and result in different grain orientations for different growth directions. With the runs in this section we wanted to show that the interface shape might have a significant influence on the grain evolution.

Because the heat flux over the wall is only partly compensated by the adopted heat flux at bottom the growth velocities in the three runs are not exactly the same. In particular, it takes 28,35 , and $22 \mathrm{~min}$ to reach the $80 \%$ solidification of silicon for the case of flat, convex and concave interface shape, respectively. However, the input data for grain boundary orientations do not depend on growth velocity nor temperature gradient, and therefore this has no effect on the results of grain evolution.

\subsection{Comparison with experiment}

Mangelinck-Noël et al. have developed a method of in-situ observation of silicon solidification by means of $\mathrm{X}$-ray synchrotron radiation [1]. This enables to track the crystal/melt interface during growth. Afterwards, this can be combined with the grain orientation analysis using electron beam scattering diffraction (EBSD). In this paper we compute the evolution of grains as observed in 
an experiment by Riberi-Béridot et al. [9]. The cooling rate was $\mathcal{R}=2 \mathrm{~K} / \mathrm{min}$ and the measured growth rate $v_{n}=23 \mu \mathrm{m} / \mathrm{s}$. Thus, the temperature gradient in the melt can be estimated as $G_{T}^{m}=14.5 \mathrm{~K} / \mathrm{m}$ (assuming $G_{T}^{m}=\mathcal{R} / v_{n}$ ).

Fig. 5(a) shows the EBSD map in the x-direction corresponding to the growth direction with interface lines (in yellow) from the in-situ observation. In the simulation, we consider a part of the experimental domain as indicated by the dashed orange lines in Fig. 5(a). The experimental sample has a ragged boundary because the melt did not continuously attached the curcible wall during the experiment. In the simulation the melt do attach always the domain wall. We also add a further grain on the left side (see (Fig. 5(B)) to demonstrate the influence of the interface shape, which is concave near the walls (see yellow lines in Fig. 5(A)). The computation domain is $16 \times 2.8 \mathrm{~mm}^{2}$ discretized by a uniform grid of $\Delta x=25 \mu \mathrm{m}$. For the grain boundary between grains (2) and (3) as well as for (2) and (4) we set $\alpha_{\text {grain }}$ similar to the observed tilting in the experiment. The value is slightly varying with the interface shape. For the grain boundary between (3) and (4), i.e. the $\Sigma 3(111)$ boundary, we fix the value according to the experiment: $\alpha_{\text {grain }}=25^{\circ}$. For the boundary between the artificially introduced grain (1) and (2), we applied 2D phase field simulations as described in [34]. In Fig. 6 the results for different interface orientations are shown. It can be clearly seen that the angle of the grain boundary depends on the inclination of the interface. We set the values computed for the various inclinations of the interface.

The probabilities for twinning on the grains (3) and (4) are estimated from the lengths of the grain boundary between twinning events. The probability has to be given for the node distance $\Delta x=25 \mu \mathrm{m}$, i.e. for every update. It was estimated to be $P_{\text {update }}=0.01875$ and $P_{\text {update }}=0.0575$ for twinning on grain number (3) and (4), respectively. The probability to have a twin after a distance $x$ is given by $1-\left(1-P_{\text {update }}\right)^{x / \Delta x}$. It is $0.3,0.4$, and 0.6 for the twins on grain (3) and 0.25 and 0.4 for the twins on (4). For comparison we convert the probabilities $P_{\text {update }}$ into those for a layer on a $\{111\}$ plane (layer distance is $d=3.15 \times 10^{-10} \mathrm{~m}$ ). Please note that the $\{111\}$ plane has an angle of $30^{\circ}$ with the growth direction and one layer in growth direction means a $\Delta x=$ $2.7 \times 10^{-10} \mathrm{~m}$. Thus, we obtain $\mathcal{P}_{\text {twin }}=2.1 \times 10^{-7}$ and $\mathcal{P}_{\text {twin }}=6.5 \times 10^{-7}$ for twinning on grain (3) and grain (4), respectively. These probabilities correspond to an undercooling of about $0.6 \mathrm{~K}$ according to the model of twin nucleation by Lin and Lan [29]. The same value was obtained for the undercooling in a facet/facet grain [34].

In the simulation we impose a similar temperature field as in the experiment and in particular a similar interface shape as in the experiment is computed. In order to get the interface shapes, we apply a heat flux $j_{\text {left }}=0.15 j$ at the left boundary, where $j$ is the heat flux at bottom (see Eq. 8). At the right hand side an adiabatic boundary condition is set as this boundary corresponds to position in the middle of the sample with nearly no curvature of the interface.

The evolution of the grain structure at different solidification stages is illustrated in Fig. 7(a-c). Please note that the colours of the grains are according to their numbering and do not reflect the orientation as in Fig. 7(d), where the 


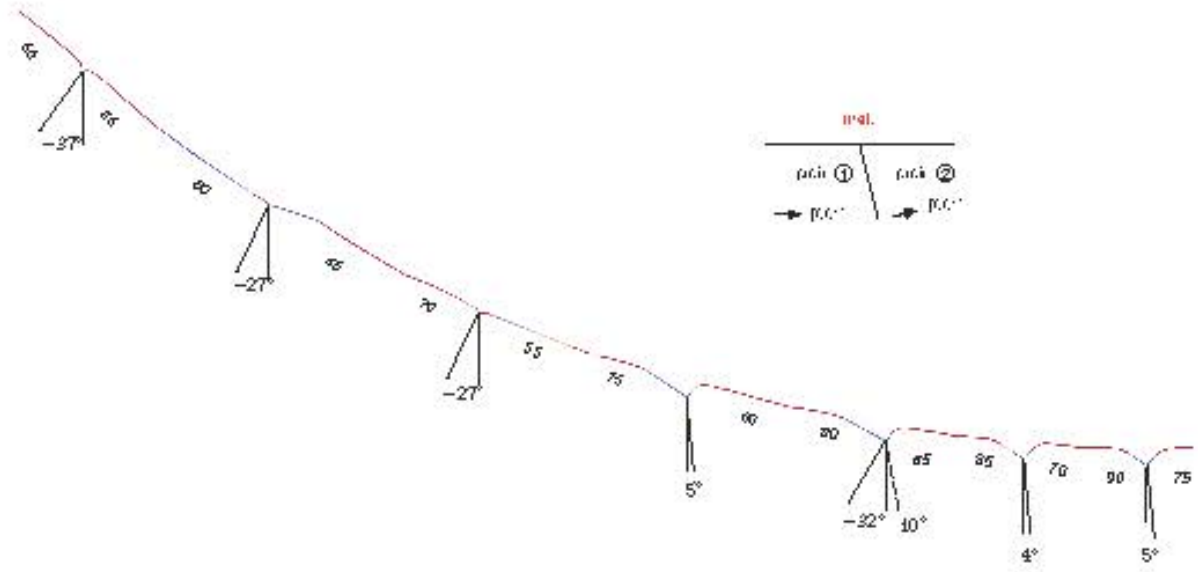

Figure 6: Results of the phase field calculations for the grain boundary between grain 0 and (2) for different orientations of the melt/solid interface. The colours at the interface represent the temperature (red: $1685 \mathrm{~K}$, blue underoooled). The angles with respect to the main growth direction are given.

colour represents the orientation along the growth direction. The white dash line shows the initial interface position and the grains grow upward. As shown in Fig. 7(a) and (b), we could also track the variation of the crystal/melt interface shape. The concave interface shapes are similar with the experimental ones, al though the curvature in the simulation is lower than that in the experiment. In addition, the final grain structure obtained in the computations is in good agreement with the experimental results of Riberi-Béridot et al. [9]. The occurrence frequency of twin nucleation is slightly higher than that in the experiment. Please note that twin nucleation is a statistical process and it cannot expected that the experimental result is exactly reproduced in the simulation. It is useful to compare statistical parameters, like grain orientation distribution. Since our simulation is based on $2 \mathrm{D}$, it is unreasonable to compare the average grain size. Average grain size often gets from the statistics from the horizontal wafers. Here, we can compare the grain orientation distribution. We notice that Grain 2, 3 and 4 own unique orientation and the area fraction of them evolve differently along with the growth height in the growth direction. As show in Fig. 8, the relative error of area fraction for Grain (2), (3), and (4) between experiment and simulation at the end of solidification is $0.2 \%, 3.6 \%$ and $4.9 \%$, respectively.

The grain boundary between grains (3) and (4) is a coherent $\Sigma 3(111)$ boundary. It can be described as a combined twist and tilt boundary with a transformation matrix ( $T$ matrix) of $T_{\langle 111\rangle, 146^{\circ}}$ (see [35]). The grain boundaries between grains 

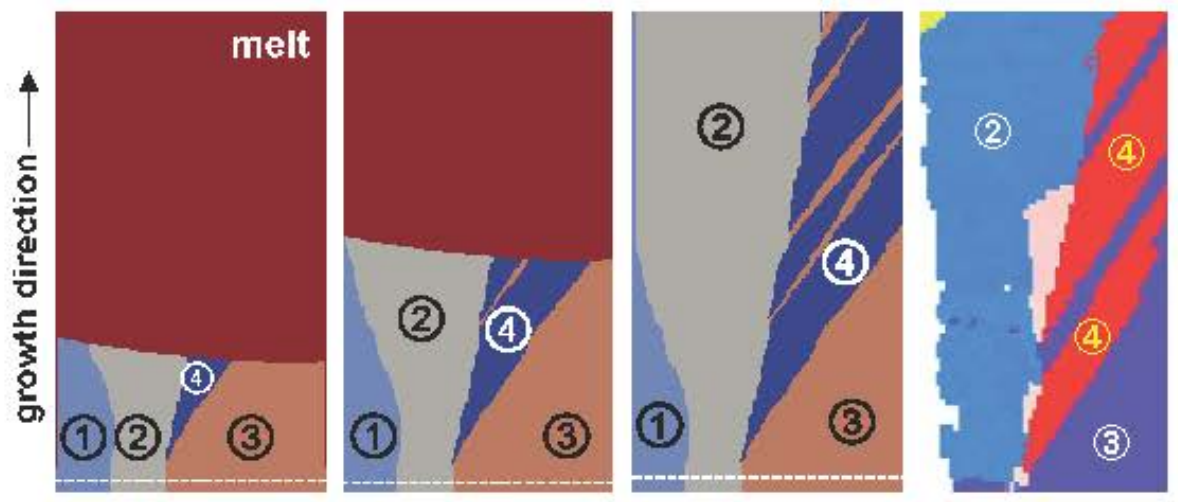

Figure r: Results of grain structure during the solidification process. Numerical calculations at different stages of solidification: (a) $25 \%$, (b) $50 \%$ and (c) $100 \%$, and (d) experimental result of Riberi et 2l. [9].

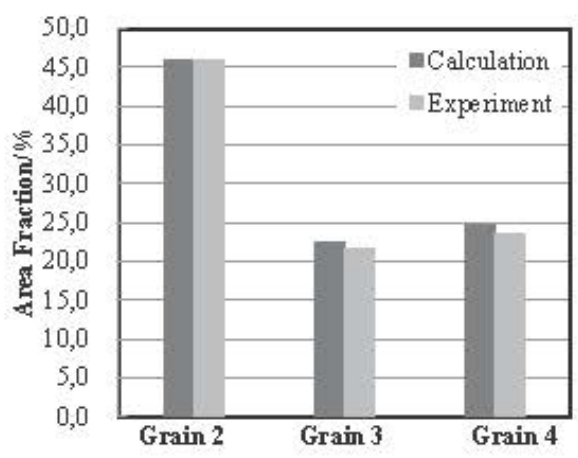

Figure 8: Area fraction of different grains at the end of solidification.

(2) and (3) as well as between (2) and (4) are not coherent, which is reflected in slightly varying orientation of the grain boundaries. This effect is not yet included in our cellular automata setup.

The raw/processed data, required to reproduce these findings cannot be shared at this time due to technical or time limitations.

\section{Conclusions}

We established a 2D model to calculate the evolution of grains based on input data from the microscopic scale simulations. In particular, the input data consists of the GB orientations with respect to the crystal/melt interface normal for every combination of GBs. It is shown that the interface shape significantly influences the grain evolution during the solidification process. In the case considered in this paper the GBs are almost straight for the flat interface shape, 
while those gradually change with the variation of the interface normal for the convex and concave interface shapes. Nucleation of twin seeds is also investigated and compared with the experimental results. The calculated crystal $/ \mathrm{melt}$ interface shape is similar to the experimental one. Moreover, the calculated grain morphology and orientation of GBs correspond with the observation in the experiments. The more input data for the CA model will be obtained by microscopic or atomistic calculations the more predective the $\mathrm{CA}$ model can be. In the future larger systems will be investigated, which will allow to make statistics on average grain size and orientation distributions.

\section{Acknowledgements}

X.F. Qi gratefully acknowledge the China Scholarship Council for financing a one year of stay at the Leibniz Institute for Crystal Growth (IKZ). The authors would like to thank the group of Ulrich Rüde at the University NürnbergErlangen for providing the WaLBErla software and for their support. Part of this work was funded by the ANR Project CrySaLID (N_ANR-14-CE05-004601)

[1] A. Tandjaoui, N. Mangelinck-Noël, G. Reinhart, J.-J. Furter, B. Billia, T. Lafford, J. Baruchel, X. Guichard, Real time observation of the directional solidification of multicrystalline silicon: X-ray imaging characterization, Energy Procedia 27 (2012) 82-87. doi:10.1016/j.egypro.2012.07.033.

[2] K. Fujiwara, Grain Growth in the Melt, Vol. IB, Elsevier, 2015, pp. 723748.

[3] G. Cantù, A. Popescu, W. Miller, Grain growth of silicon, Acta Mater. 60 (2012) 6755-6761. doi:10.1016/j.actamat.2012.08.048.

[4] P. Chen, Y. L. Tsai, C. W. Lan, Phase field modeling of growth competition of silicon grains, Acta Mater. 56 (2008) 4114-4122. doi:10.1016/j.jcrysgro.2017.06.016.

[5] K. Fujiwara, Y. Obinata, T. Ujihara, N. Usami, G. Sazaki, K. Nakajima, Grain growth behaviors of polycrystalline silicon during melt growth processes, J. Crystal Growth 266 (2004) 441-448. doi:10.1016/j.jcrysgro.2004.03.008.

[6] T. Duffar, A. Nadri, The grain-grain-liquid triple phase line during solidification of multi-crystalline silicon, C. R. Physique 14 (2-3) (2013) 185-191. doi:10.1016/j.crhy.2012.12.003.

[7] W. Miller, A. Popescu, G. Cantù, Solidification of multicrystalline silicon - simulation of micro structures, J. Crystal Growth 385 (2014) 127-133. doi:0.1016/j.jcrysgro.2013.01.044. 
[8] H. K. Lin, M. C. Wu, C. C. Chen, C. W. Lan, Evolution of grain structures during directional solidification of silicon wafers, J. Crystal Growth 439 (2016) 40-46. doi:10.1016/j.jcrysgro.2015.12.050.

[9] T. Riberi-Béridot, N. Mangelinck-Noël, A. Tandjaoui, G. Reinhart, B. Billia, T. Lafford, J. Baruchel, L. Barrallier, On the impact of twinning on the formation of the grain structure of multi-crystalline silicon for photovoltaic applications during directional solidification, J. Crystal Growth 418 (2015) 38-44. doi:10.1016/j.jcrysgro.2015.02.024.

[10] R. R. Prakash, K. Jiptner, J. Chen, Y. Miyamura, H. Harada, T. Sekiguchi, Grain boundary interactions in multicrystalline silicon grown from small randomly oriented seeds, Applied Phys. Express 8 (3) (2015) 035502-1-4. doi:10.7567/APEX.8.035502.

[11] J. A. Warren, R. Kobayashi, A. E. Lobkovsky, W. C. Carter, Extending phase field models of solidification to polycrystalline materials, Acta Mater. 51 (2003) 6035. doi:10.1016/S1359-6454(03)00388-4.

[12] A. Nadri, Y. Duterrail-Courvat, T. Duffar, Two-dimensional numerical modeling of grain structure in multi-crystalline silicon ingot, J. Crystal Growth 385 (2014) 16-21. doi:10.1016/j.jcrysgro.2013.04.013.

[13] B. Wu, R. Clark, Influence of inclusion on nucleation of silicon casting for photovoltaic (PV) application, J. Crystal Growth 318 (2011) 200-207. doi:10.1016/j.jcrysgro.2010.10.079.

[14] K. G. F. Janssens, An introductory review of cellular automata modeling of moving grain boundaries in polycrystalline materials, Math. Comput. Simul. 80 (2010) 1361-1381. doi:10.1016/j.matcom.2009.02.011.

[15] Y. Natsume, K. Ohsasa, Three-dimensional cellular automaton model for the prediction of microsegregation in solidification grain structures, ISIJ International 54 (2014) 415-421. doi:10.2355/isijinternational.54.415.

[16] M. Rappaz, C. A. Gandin, Probabilistic modelling of microstructure formation in solidification processes, Acta metall. mater. 41 (2) (1993) 345-360. doi:10.1016/0956-7151(93)90065-Z.

[17] Q. Lian, W. Liu, R. Li, W. Yan, C. Liu, Y. Zhang, L. Wang, H. Chen, Numerical simulation of multi-crystalline silicon crystal growth using a macromicro coupled method during the directional solidification process, Appl. Sci. 7 (21) (2017) 21-1-13. doi:doi:10.3390/app7010021.

[18] A. R. A. Dezfoli, A. K. S. Weng-Sing Hwang and, J. Augusto, Y. S. Huang, S. Tzeng, 3d numerical study of coupled crystallization and carbon segregation during multi-crystalline silicon ingot solidification, Mater. Sci. Semicond. Proc. 59 (2017) 76-86. doi:10.1016/j.mssp.2016.11.043. 
[19] A. R. A. Dezfoli, Weng-SingHwang, J. Augusto, A. K. Shukura, ShikaiTzeng, Modeling of poly-crystalline silicon ingot crystallization during casting and theoretical suggestion for ingot quality improvement, Mater. Sci. Semicond. Proc. 53 (2016) 36-46. doi:10.1016/j.mssp.2016.05.013.

[20] R. Kobayashi, J. A. Warren, W. C. Carter, Vector-valued phase field model for crystallization and grain boundary formation, Physica D 119 (1998) $415-423$.

[21] O. Weinstein, S. Brandon, Dynamics of partially faceted melt/crystal interfaces I: computational approach and single step-source calculation, J. Crystal Growth 268 (2004) 299-319. doi:10.1016/j.jcrysgro.2004.04.108.

[22] I. Rasin, Numerical simulation of microstructure of the gesi alloy, Ph.D. thesis, Brandenburgische Technische Universität Cottbus (2006).

[23] I. Rasin, S. Succi, W. Miller, A multi-relaxation lattice kinetic method for passive scalar diffusion, J. Comput. Phys. 206 (2) (2005) 453-462. doi:doi:10.1016/j.jcp.2004.12.010.

[24] Y. Inatomi, F. Onishi, K. Nagashio, K. Kuribayashi, Density and thermal conductivity measurements for silicon melt by electromagnetic levitation under a static magnetic field, Int. J. Thermophys. 28 (1) (2007) 44-59.

[25] A. Virzi, Computer modelling of heat transfer in Czochralski silicon crystal growth, J. Crystal Growth 112 (1991) 699-722.

[26] K.-H. Hellwege, O. Madelung (Eds.), Landolt-Börnstein, Vol. 17, SpringerVerlag, Berlin, 1984.

[27] M. Watanabe, Talk at ICCG-16, Beijing (2010).

[28] T. Duffar, A. Nadri, On the twinning occurrence in bulk semiconductor crystal growth, Scripta Mater. 62 (12) (2010) 955-960. doi:10.1016/j.scriptamat.2010.02.034.

[29] H. K. Lin, C. W. Lan, Revisiting the twinning mechanism in directional solidification of multi-crystalline silicon sheet, Acta Mater. 131 (2017) 1-10. doi:10.1016/j.actamat.2017.03.077.

[30] F. Schornbaum, U. Rüde, Massively parallel algorithms for the lattice boltzmann method on nonuniform grids read more: https://epubs.siam.org/doi/10.1137/15m1035240, SIAM J. Sci. Comput. 38 (2) (2016) C96-C126. doi:10.1137/15M1035240.

[31] C. Feichtinger, S. Donath, H. Köstler, J. Götz, U. Rüde, Walberla: Hpc software design for computational engineering simulations, Journal of Computational Science 2 (2) (2011) 105-112. 
[32] G. Williams, R. E. Reusser, Heat transfer in silicon Czochralski crystal growth, J. Crystal Growth 64 (3) (1983) 448-460.

[33] H. Kobatake, H. Fukuyama, I. Minato, T. Tsukada, S. Awaji, Noncontact modulated laser calorimetry of liquid silicon in a static magnetic field, J. Appl. Phys. 104 (054901-1-8).

[34] W. Miller, A. Popescu, Micro structures in the grain evolution during solidification of silicon: Phase field calculations, Acta Mater. 140 (2017) 1-9. doi:10.1016/j.actamat.2017.08.025.

[35] J. W. Jhang, T. Jain, H. K. Lin, C.-W. Lan, Possible twinning operations during directional solidification of multi-crystalline silicon, Crystal Growth \& Design 18 (2018) 2518-2524. doi:10.1021/acs.cgd.8b00115. 